\title{
Proposal for a Candidate Core Set of Fitness and Strength Tests for Patients with Childhood or Adult Idiopathic Inflammatory Myopathies
}

\author{
Djamilla K.D. van der Stap, Lisa G. Rider, Helene Alexanderson, Adam M. Huber, \\ Bruno Gualano, Patrick Gordon, Janjaap van der Net, Pernille Mathiesen, Liam G. Johnson, \\ Floranne C. Ernste, Brian M. Feldman, Kristin M. Houghton, Davinder Singh-Grewal, \\ Abraham Garcia Kutzbach, Li Alemo Munters, and Tim Takken, on behalf of the \\ International Myositis Assessment and Clinical Studies Group
}

\begin{abstract}
Objective. Currently there are no evidence-based recommendations regarding fitness and strength tests for patients with childhood or adult idiopathic inflammatory myopathies (IIM). This hinders clinicians and researchers in choosing the appropriate fitness- or muscle strength-related outcome measures for these patients. Through a Delphi survey, we aimed to identify a candidate core set of fitness and strength tests for children and adults with IIM.

Methods. Fifteen experts participated in a Delphi survey that consisted of 5 stages to achieve a consensus. Using an extensive search of published literature and through the work of experts, a candidate core set based on expert opinion and clinimetrics properties was developed. Members of the International Myositis Assessment and Clinical Studies Group were invited to review this candidate core set during the final stage, which led to a final candidate core set.

Results. A core set of fitness- and strength-related outcome measures was identified for children and adults with IIM. For both children and adults, different tests were identified and selected for maximal aerobic fitness, submaximal aerobic fitness, anaerobic fitness, muscle strength tests, and muscle function tests.

Conclusion. The core set of fitness- and strength-related outcome measures provided by this expert consensus process will assist practitioners and researchers in deciding which tests to use in patients with IIM. This will improve the uniformity of fitness and strength tests across studies, thereby facilitating the comparison of study results and therapeutic exercise program outcomes among patients with IIM. (First Release November 15 2015; J Rheumatol 2016;43:169-76; doi:10.3899/jrheum.150270)
\end{abstract}

Key Indexing Terms:

EXERCISE FITNESS MYOSITIS OUTCOME MEASURES INSTRUMENTS

\footnotetext{
From the Faculty of Human Movement Sciences, Free University, Amsterdam; Child Development and Exercise Center, Wilhelmina Children's Hospital, University Medical Center Utrecht, Utrecht, the Netherlands; Environmental Autoimmunity Group, National Institute of Environmental Health Sciences, National Institutes of Health, Bethesda, Maryland; Department of Internal Medicine, Division of Rheumatology, Mayo Clinic in Rochester, Rochester, Minnesota; Division of Rheumatology and Immunology, Department of Medicine, Vanderbilt University, Nashville, Tennessee, USA; Department of Neurobiology, Care Science and Society; Division of Physical Therapy, Karolinska Institutet; Physical Therapy Clinic, Orthopedic/Rheumatology Unit, Karolinska University Hospital, Stockholm, Sweden; Izaak Walton Killam (IWK) Health Centre and Dalhousie University, Halifax, Nova Scotia; Department of Pediatrics, and Department of Medicine, and the Institute of Health Policy, Management and Evaluation, University of Toronto; Division of Rheumatology, The Hospital for Sick Children, Toronto; British Columbia's Children's Hospital; University of British Columbia, Vancouver, British Columbia, Canada; University of Sao Paulo, Sao Paulo, Brazil; Department of Rheumatology, King's College Hospital, London, UK; Paediatric Rheumatology Clinic, Paediatric Department, Rigshospitalet, Copenhagen, Denmark; Institute of Sport, Exercise and Active Living (ISEAL), College of Sport and Exercise Science, Victoria University; The Florey Institute of Neuroscience and Mental Health,
}

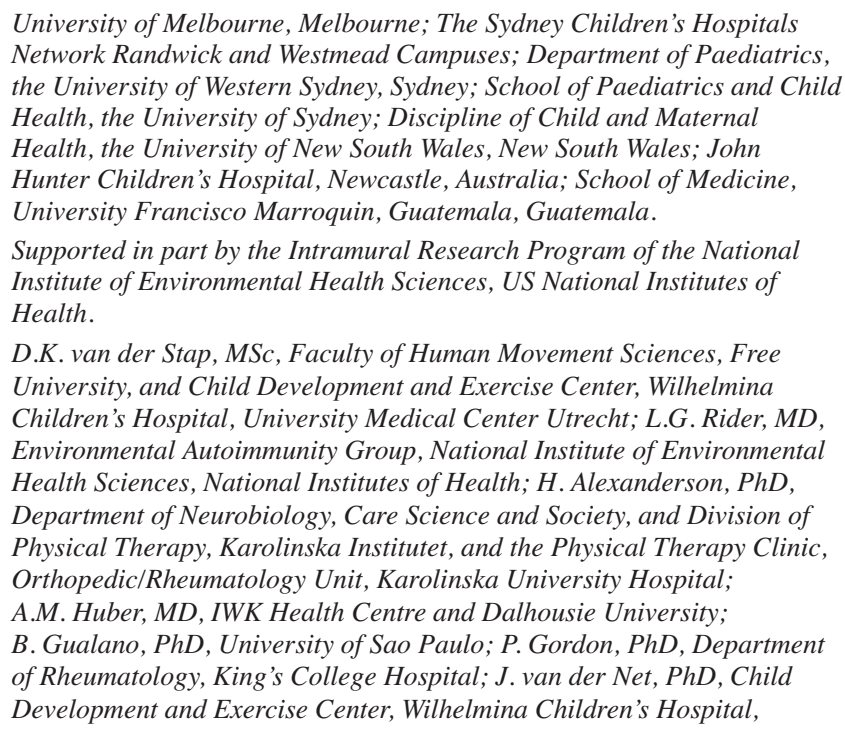

University of Melbourne, Melbourne; The Sydney Children's Hospitals Network Randwick and Westmead Campuses; Department of Paediatrics, the University of Western Sydney, Sydney; School of Paediatrics and Child Health, the University of Sydney; Discipline of Child and Maternal Health, the University of New South Wales, New South Wales; John Hunter Children's Hospital, Newcastle, Australia; School of Medicine, University Francisco Marroquin, Guatemala, Guatemala.

Supported in part by the Intramural Research Program of the National Institute of Environmental Health Sciences, US National Institutes of Health.

D.K. van der Stap, MSc, Faculty of Human Movement Sciences, Free University, and Child Development and Exercise Center, Wilhelmina Children's Hospital, University Medical Center Utrecht; L.G. Rider, MD, Environmental Autoimmunity Group, National Institute of Environmental Health Sciences, National Institutes of Health; H. Alexanderson, PhD, Department of Neurobiology, Care Science and Society, and Division of Physical Therapy, Karolinska Institutet, and the Physical Therapy Clinic, Orthopedic/Rheumatology Unit, Karolinska University Hospital; A.M. Huber, MD, IWK Health Centre and Dalhousie University; B. Gualano, PhD, University of Sao Paulo; P. Gordon, PhD, Department of Rheumatology, King's College Hospital; J. van der Net, PhD, Child Development and Exercise Center, Wilhelmina Children's Hospital, 
University Medical Center Utrecht; P. Mathiesen, PhD, Paediatric Rheumatology Clinic, Paediatric Department, Rigshospitalet; L.G. Johnson, PhD, ISEAL, College of Sport and Exercise Science, Victoria University, and the Florey Institute of Neuroscience and Mental Health, University of Melbourne; F.C. Ernste, MD, Department of Internal Medicine, Division of Rheumatology, Mayo Clinic in Rochester; B.M. Feldman, MD, Department of Pediatrics, and Department of Medicine, and the Institute of Health Policy, Management and Evaluation, University of Toronto, and Division of Rheumatology, The Hospital for Sick Children; K.M. Houghton, MD, British Columbia's Children's Hospital, and The University of British Columbia; D. Singh-Grewal, PhD, the Sydney Children's Hospitals Network Randwick and Westmead Campuses, and School of Paediatrics and Child Health, University of Sydney, and Discipline of Child and Maternal Health, University of New South Wales, and Department of Paediatrics, University of Western Sydney, and John Hunter Children's Hospital; A. Garcia Kutzbach, MD, Director of the Postgraduate Program of Rheumatology AGAR, and Professor of Medicine and History of Medicine, School of Medicine University Francisco Marroquin, and Member of the Executive Committee Hospital Herrera Llerandi Guatemala; L. Alemo Munters, PhD, Physical Therapy Clinic, Orthopedic/Rheumatology Unit, Karolinska University Hospital, and Division of Rheumatology and Immunology, Department of Medicine, Vanderbilt University; T. Takken, PhD, Child Development and Exercise Center, Wilhelmina Children's Hospital, University Medical Center Utrecht.

Address correspondence to Dr. T. Takken, Child Development and Exercise Center, Wilhelmina Children's Hospital, University Medical Center Utrecht, KB.02.056.0, P.O. Box 85090, 3508 AB Utrecht, the Netherlands. E-mail:t.takken@umcutrecht.nl

Accepted for publication September 21, 2015.

Childhood and adult idiopathic inflammatory myopathies (IIM) are rare systemic autoimmune diseases characterized by chronic muscle inflammation and weakness ${ }^{1}$. Patients with the subtypes of IIM - juvenile dermatomyositis (JDM), juvenile polymyositis, adult dermatomyositis, adult polymyositis, and inclusion body myositis - frequently experience anaerobic and aerobic exercise intolerance and fatigue $^{2,3,4}$, and may be limited in their daily physical functioning, which can lead to a poorer quality of life $\mathrm{e}^{5}$. This has led to an exploration of interventions such as exercise training programs ${ }^{6}$. To assess the clinical status of IIM and to quantify changes in physical functioning over time, validated fitness and strength tests are essential.

Fitness tests can be divided into 3 general categories: maximal aerobic fitness tests, submaximal aerobic fitness tests, and anaerobic fitness tests. Maximal aerobic fitness tests determine the maximal oxygen uptake $\left(\mathrm{VO}_{2 \max }\right)$. Submaximal aerobic fitness tests predict aerobic fitness using a submaximal exercise protocol (i.e., an exercise test that does not require the participant's maximal effort $)^{7,8}$. Anaerobic fitness tests measure anaerobic performance and/or estimate the capacity of anaerobic energy pathways (e.g., phosphorylcreatine system and anaerobic glycolysis) during short duration, high-intensity maximal exercise (usually $<30 \mathrm{~s})^{9}$. Muscle strength tests measure neuromuscular performance and can be measured with either static or dynamic muscle contractions. The neuromuscular performance measured is specific to the muscle group that is tested; for a comprehensive assessment of muscle strength, several major muscle groups must be assessed. A person's maximum strength for a given muscle group corresponds to the maximum force that they can generate. For muscle strength tests, a distinction was made between tests that explicitly measure muscle force generation capacity, referred to as muscle strength tests, and those that measure performance-based functional capacity, referred to as muscle function tests.

While fitness and strength testing over time can provide a quantitative assessment of the improvement or decline in the physical condition and strength of the patient with IIM, no clear recommendations are currently available for clinicians and researchers regarding which fitness or strength tests should be used in patients with IIM $^{1}$. As a result, a large variety of outcome measures have been used to evaluate the fitness and muscle strength of patients with IIM; however, most of these instruments have not been validated for this patient group ${ }^{1}$. With a core set of fitness- and strength-related outcome measures, the uniformity of fitness and strength tests across studies would improve, thereby facilitating the comparison of study results, allowing for a better comparison of the effects of therapeutic exercise programs.

The aim of this study was to provide a list of evidence-informed fitness- and strength-related outcome measures for patients with IIM, and thus facilitate clinicians and researchers to make better decisions about which tests to use for this patient group. This core set of fitness and strength tests will serve as a candidate core set and will be a basis for future research.

\section{MATERIALS AND METHODS}

Design. The Delphi survey method was used in our study. The Delphi method has been developed to assess opinions and judgments, rather than objective facts, to reach consensus among a group of individuals ${ }^{10}$. With the use of questionnaires, a panel of informed individuals, subsequently called experts, was asked to give feedback about a particular issue to achieve a group consensus. Experts were selected based on their activities within the International Myositis Assessment and Clinical Studies Group (IMACS) or because of their expertise in this research area.

During stage 1 of the Delphi survey, literature was searched for fitness- and muscle strength-related outcome measures that were used in IIM. PubMed and Google Scholar were searched up to April 2013 using the following search terms: "physical fitness," "exercise testing," "exercise," "exercise capacity," "exercise tolerance," "muscle strength," "muscle force," "dermatomyositis," and "myositis." All articles that were available at the time of the search and that matched the inclusion criteria were included in this Delphi survey. Studies were included if (1) the study population of the articles consisted of patients with IIM and (2) the studies included an examination of a fitness and/or strength test. No other inclusion and exclusion criteria were applied. Further, reference lists of all the selected articles were searched for additional studies. The outcome measures were listed and categorized as follows: (1) maximal aerobic fitness tests, (2) submaximal aerobic fitness tests, (3) anaerobic fitness tests, (4) muscle strength tests, and (5) muscle function tests. Afterward, the experts were asked to list additional tests from their clinical practice or from the unpublished or published literature that were not yet listed to make the list as complete as possible.

In stage 2, the experts were asked to rate the completed list of fitness and muscle strength tests that were identified in the first Delphi stage. All the tests were rated separately for children and adults. All experts rated each

Personal non-commercial use only. The Journal of Rheumatology Copyright (C) 2016. All rights reserved 
outcome measure on a 10-point scale for 4 different topics (i.e., safety, suitability, user friendliness, and overall rating). These 4 topics were chosen based on their previous use in a comparable Delphi of fitness outcomes for children with cerebral palsy ${ }^{11}$. Moreover, the experts were asked for additional information about the clinimetric properties of the listed tests. When a test was not studied in the IIM population, clinimetric properties were noted as unavailable.

A draft of the core set was identified in stage 3 of the Delphi survey. For each category, a test or several tests were selected based on expert ratings (median scores were provided) and clinimetric properties of the tests collected during stages 1 and 2. This core set was presented to the experts together with the complete list of outcome measures and their ratings. Subsequently, the experts were asked whether they agreed or disagreed with each measure's inclusion in the suggested core set.

Based on the comments made by the experts during stage 3 , a final draft of the candidate core set was presented in stage 4 . The experts were asked if they agreed or disagreed with the suggested core set for both adults and children. Further, a conference call was organized to discuss the comments of the experts and to reach consensus among experts.

In stage 5 of the Delphi survey, the final draft candidate of the core set was sent out to all the IMACS members through an Internet survey to reach consensus. The IMACS members could choose to complete the questionnaire anonymously or include their name (Appendix 1 for the participants who have chosen to include their name). IMACS members were asked whether they agreed or disagreed with the tests included in the core set, or to state if they were unfamiliar with a selected test. Further, they were requested to report any important test that, in their opinion, was missing from the final draft candidate core set. Based on the outcome of the Internet survey, the final draft candidate core set was once more revised. Consensus was achieved when at least $75 \%$ of the IMACS members agreed on the inclusion of a given test in the core set; otherwise, the test was removed from the core set. This predefined cutoff score was selected as a comparable cutoff that has been used previously by the IMACS ${ }^{12}$.

Statistical analysis. The experts' rating of the fitness and strength tests during each of the Delphi stages was summarized with descriptive statistics using IBM SPSS Statistics for Windows, version 20.0.

\section{RESULTS}

Fifteen experts participated in this Delphi survey, but only 12 completed the entire survey. One expert dropped out during stage 1 because of a lack of time, while the other 2 experts, both adult physicians, dropped out during stage 2 because they felt they did not have enough expertise to score all tests. The experts' characteristics can be found in Appendix B (available from the authors on request).

Results of Delphi stage 1. In the first stage of the Delphi survey, lasting from February 1, 2013, until June 10, 2013, 22 tests were identified in the literature search. Further, the experts suggested 16 additional tests, though 7 of these tests were excluded because they did not measure an outcome of interest. See Figure 1 for a flow diagram showing the identification of the tests. For the complete list of tests identified, see Appendix C (available from the authors on request).

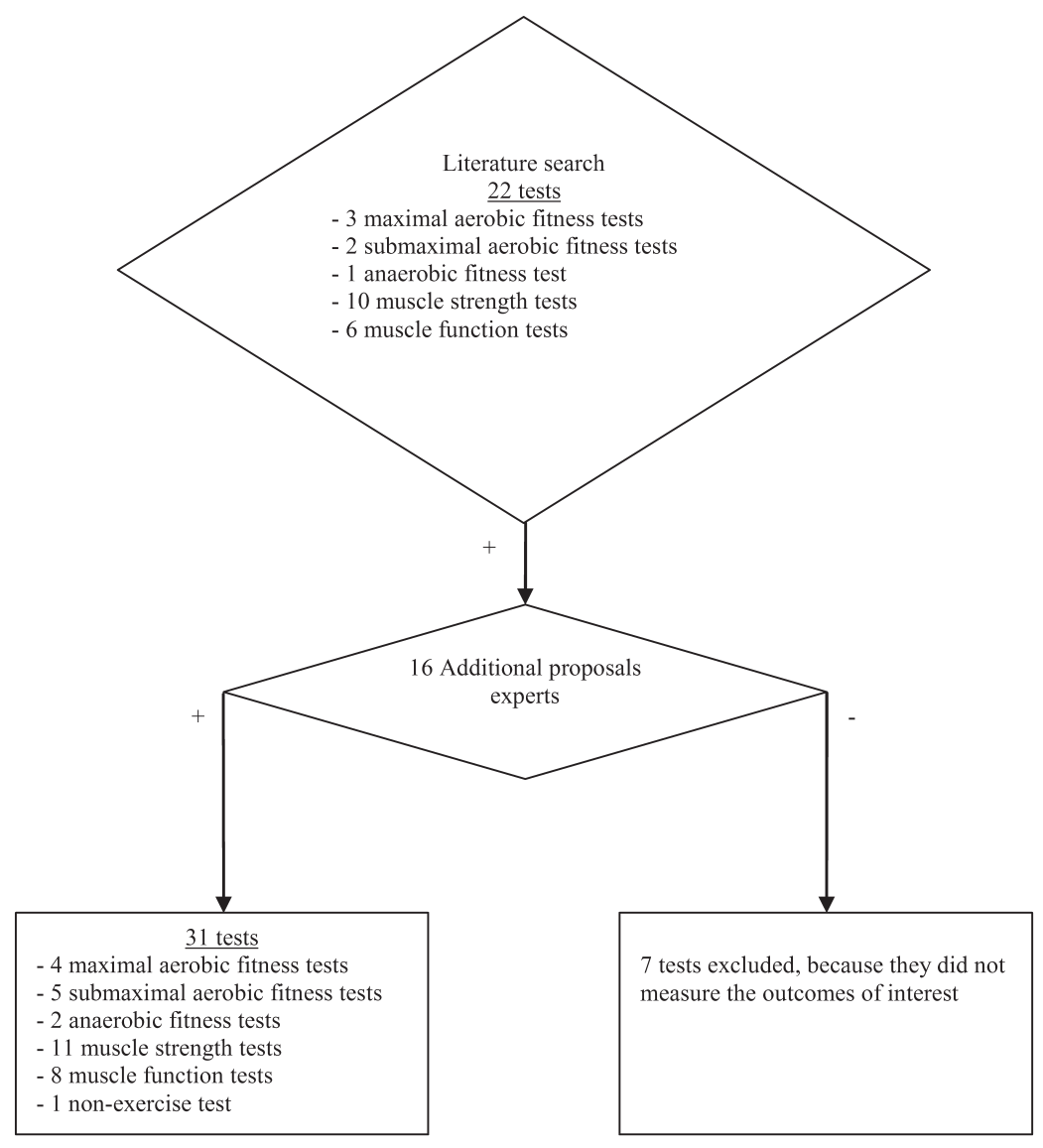

Figure 1. Flow diagram on the identification of the tests. 
For maximal aerobic fitness tests, the incremental cycle ergometer test [intraclass correlation coefficient (ICC) > 0.95] was the only one with published reliability data in $\mathrm{IIM}^{12}$. No data on reliability and/or validity of submaximal aerobic fitness tests in IIM were available. For anaerobic fitness tests, the Wingate cycle test (ICC >0.85) was the only test with published reliability data in IIM $^{12}$.

The isometric dynamometer, Manual Muscle Testing (MMT), and the $1-\mathrm{kg}$ arm lift test were the only muscle strength tests with available reliability and concurrent validity data. The isometric dynamometer as used by Stoll, et al ${ }^{13}$ showed strong and significant intra- and interobserver correlations, as well as significant and strong correlations between measurements of the left and right sides. MMT was highly correlated with total and proximal MMT scores and with the Childhood Myositis Assessment Scale (CMAS), and moderately correlated with physician's global activity, functional disability, magnetic resonance imaging (MRI), and axial and distal MMT scores, and in adults, with creatine kinase level ${ }^{1}$. The 1-kg arm lift test showed excellent test-retest reliability, and correlated inversely with serum creatine kinase ${ }^{14}$.

For several muscle function tests, reliability and concurrent validity data were available in IIM: CMAS in children $(\mathrm{ICC}=0.89$, highly correlated with the Childhood Health Assessment Questionnaire score and with MMT scores, and moderately correlated with physician-assessed global disease activity and skin activity, parent-assessed global disease severity and muscle MRI) ${ }^{15}$, the Functional Index (FI-2) for adults (ICC $=0.86-0.99$, moderately correlated with the shoulder flexion task of the preliminary revised FI and isokinetic measurements of shoulder flexion endurance) ${ }^{16}$, and the 30 -s chair stand test (excellent test-retest reliability, correlated inversely with serum creatine kinase $)^{14}$.

Results of Delphi stage 2. In the second Delphi stage, all experts were asked to rate the complete list of outcome measures (Appendix C, available from the authors on request) for safety, suitability, user friendliness, and overall rating for both children and adults. The e-mail to the experts was sent out on June 10, 2013, and the experts were given 7 weeks to complete the survey. The experts had no additional information about the clinimetric properties.

Results of Delphi stages 3 and 4. On September 3, 2013, a draft core set of fitness and strength measures was presented to the experts. The tests that made it into the draft core set were selected because they had the highest median scores in their category and/or because they had good reliability and validity data. However, because of the lack of available clinimetric properties, most tests were included based on expert's opinion rather than on reliability and validity. The median scores and interquartile ranges of the selected tests in the proposed core set are presented in Table 1. Experts got another 7 weeks to respond on the draft core set.

Based on this draft core set, a conference call was organized on November 12, 2013, with the expert panel, including the 3 experts who dropped out during previous stages. Using the comments from the third Delphi stage and from the conference call, a revised core set was developed (Table 2). In this revised core set, 2 major changes were made: the Åstrand cycle test was added in the core set for the adults, while the CMAS was removed for the adults. These changes were discussed during the conference call and consensus about these changes and the revised core set among experts was reached.

Results of the final Delphi stage. The online questionnaire was sent out on March 28, 2014, and there were 88 additional IMACS members who responded to the online questionnaire, 57 anonymously, and 31 IMACS members who chose to include their names, which are listed in the appendix of contributors (Appendix 1). Based on the consensus scores (Table 2), some tests were removed from the draft candidate core set because they did not meet the requirement of the $\geq$ $75 \%$ consensus agreement. There were no additional tests added because none of the additional tests were mentioned more than twice, which was not enough to reach consensus. A final candidate core set was made, as shown in Table 3. The final candidate core set included 5 tests for children and 6 for adults, of which the MMT, CMAS, and FI-2 have been validated and shown to be reliable in people with IIM.

\section{DISCUSSION}

Although several other groups have identified and proposed core sets of outcome measures for the IIM population $^{17,18,19,20}$, none of these efforts have specifically focused on a core set for fitness- and strength-related outcomes.

Maximal aerobic fitness tests. The modified Bruce protocol $^{2,21,22,23}$ and the incremental cycle ergometer test $\mathrm{t}^{24}$ were the 2 maximal aerobic fitness tests that were included in the core set for both adults and children after the first 4 stages of the Delphi survey. Both tests were chosen based on expert opinion rather than on their clinimetric properties. There was reliability data available for maximal cycle ergometry $^{25}$. During the final stage of the Delphi survey, there was no consensus reached about whether to use the modified Bruce protocol in children. Therefore, this test was removed from the candidate core set.

For adult patients with IIM, both the modified Bruce protocol and the incremental cycle ergometer protocol were included in the final core set. The experts decided it would be better to use the modified Bruce protocol because it makes the test more accessible for patients with IIM with reduced physical function. The incremental cycle ergometer test includes workload increases dependent on disease activity and body height with 10,15 , or 20 watts/min 24,25 . In addition, the expert panel advised a 5 watts/min increase for very weak patients.

There were no data available regarding the clinimetric properties of the (modified) Bruce protocol or the incremental

Personal non-commercial use only. The Journal of Rheumatology Copyright @ 2016 . All rights reserved. 
Table 1. Overview of tests that were presented during stage 3 of the Delphi survey. All tests were rated on a 10-point scale. Values are median (interquartile range).

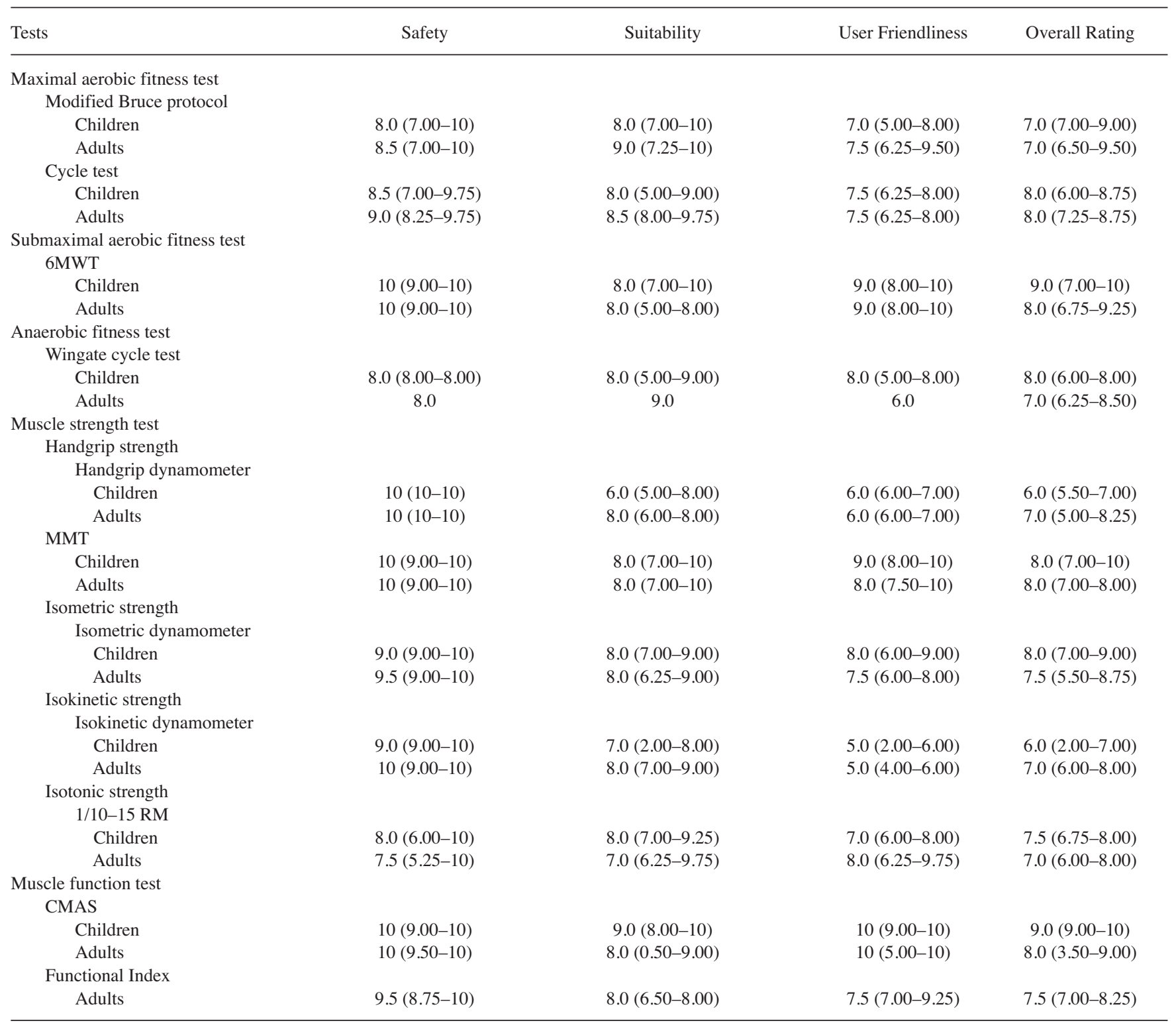

6MWT: 6-min walk test; MMT: Manual Muscle Testing; RM: repetition maximum; CMAS: Childhood Myositis Assessment Scale.

cycle ergometer test, and therefore further research is needed to validate these tests for IIM. Further research is also needed to identify maximal aerobic field tests to include in the core set.

Submaximal aerobic fitness tests. During the first 4 Delphi stages, the 6-min walk test (6MWT) was included in the core set for both children and adults. As an addition to the core set for adults, the Åstrand cycle test was also included. Both tests were included based on expert opinion rather than on clinimetric properties because these were not available. However, during the final stage of the Delphi survey, there was clearly no consensus about the use of the Åstrand cycle test in adults.
Therefore, this test was removed from the final candidate core set.

The 6MWT is a practical and simple test that is inexpensive and easy to administer, and it allows individuals to set their own pace and voluntarily stop if necessary. The 6MWT is currently one of the core outcome measures in trials involving patients with muscle disease ${ }^{26}$. All experts agreed that the 6MWT should be included in the core set. The Åstrand cycle test was initially added as a result of the feedback received in the third Delphi stage based on the clinical experience of one of the expert physical therapists. This could be an optional test for patients who have difficulty

Personal non-commercial use only. The Journal of Rheumatology Copyright @ $\ 2016$. All rights reserved. 
Table 2. Results of the online questionnaire among all IMACS members during stage 5 of the Delphi survey. The percentages provided in this table reflect the percentage of the IMACS members who agree on the given test of the core set. Values are $\%(n)$

\begin{tabular}{|c|c|c|}
\hline Tests & Children & Adults \\
\hline \multicolumn{3}{|l|}{ Maximal aerobic fitness test } \\
\hline Modified Bruce protocol, treadmill & $58.3(12)$ & $87.5(16)$ \\
\hline Incremental exercise test, cycle ergometer & $75.0(14)$ & $83.3(18)$ \\
\hline \multicolumn{3}{|c|}{ Submaximal aerobic fitness test } \\
\hline 6MWT & $85.7(21)$ & $89.3(28)$ \\
\hline Åstrand cycle test* & NA & $20.0(15)$ \\
\hline \multicolumn{3}{|l|}{ Anaerobic fitness test } \\
\hline Wingate cycle test & $55.6(9)$ & $61.5(13)$ \\
\hline \multicolumn{3}{|l|}{ Muscle strength test } \\
\hline \multicolumn{3}{|l|}{ Handgrip strength } \\
\hline Handgrip dynamometer & $82.4(17)$ & $81.5(27)$ \\
\hline MMT & $95.0(20)$ & $96.6(29)$ \\
\hline \multicolumn{3}{|l|}{ Isometric strength } \\
\hline Isometric dynamometer & $46.2(13)$ & $54.5(22)$ \\
\hline \multicolumn{3}{|l|}{ Isokinetic strength } \\
\hline Isokinetic dynamometer & $41.7(12)$ & $45.5(22)$ \\
\hline \multicolumn{3}{|l|}{ Isotonic strength } \\
\hline 1/10-15 RM & $53.8(13)$ & $54.5(22)$ \\
\hline \multicolumn{3}{|l|}{ Muscle function test } \\
\hline CMAS & $95.0(20)$ & NA \\
\hline Functional Index & NA & $95.0(20)$ \\
\hline
\end{tabular}

* Test was added by the experts during the first stage. IMACS: International Myositis Assessment and Clinical Studies Group; 6MWT: 6-min walk test; MMT: Manual Muscle Testing; RM: repetition maximum; CMAS: Childhood Myositis Assessment Scale; NA: not applicable.

Table 3. Final candidate core set of fitness and muscle strength tests.

\begin{tabular}{lcc}
\hline Tests & Children & Adults \\
\hline Maximal aerobic fitness test & & \\
$\quad \begin{array}{l}\text { Modified Bruce protocol, treadmill } \\
\text { Incremental exercise test, cycle ergometer }\end{array}$ & + & + \\
Submaximal aerobic fitness test & + & + \\
6MWT & & + \\
Muscle strength test & & + \\
Handgrip strength & + & + \\
$\quad$ Handgrip dynamometer & + & + \\
MMT & & + \\
Muscle function test & + & + \\
CMAS & - & + \\
Functional Index-2 & & \\
\hline
\end{tabular}

6MWT: 6-min walk test; MMT: Manual Muscle Testing; CMAS: Childhood Myositis Assessment Scale.

walking. The Åstrand cycle test was added for adults only because it has been found to have large measurement errors in children ${ }^{27}$. Even though this test is removed from the final candidate core set, it could still be recommended in adult patients who have difficulty walking. Further research is needed to validate these tests for IIM.

Anaerobic fitness tests. Only the Wingate cycle test was included in the draft candidate core set to measure anaerobic fitness $^{3,25}$. There were only 2 anaerobic fitness tests identified in the first Delphi stage, and the clinimetric properties of the Wingate cycle test in JDM have been published ${ }^{25}$. Therefore, based on expert opinion and clinimetric properties, the Wingate cycle test was initially included. However, only $55.6 \%$ of the IMACS members agreed that this test should be in the core set for children, and only $61.5 \%$ of the IMACS members agreed that this test should be included in the core set for adults. Unfortunately, no other alternative tests of anaerobic fitness have been identified, and therefore the final candidate core set does not include an anaerobic fitness test. There is also no field-based anaerobic fitness test available yet for the IIM population. Future studies should investigate the clinimetric properties of a field-based anaerobic fitness test, such as the muscle power sprint test ${ }^{9}$, to determine its potential use as a fitness test for the IIM population.

Muscle strength tests. Based on the first 4 stages of the Delphi survey, handgrip strength ${ }^{21,22,23,28,29,30,31,32}$ and MMT $^{29,32-41}$ were identified as common measures of muscle strength in both adults and children. The IMACS members agreed on these 2 tests for both children and adults because consensus scores of at least $75 \%$ were reached. For a better understanding of patients' muscle strength, measurements of isometric, isokinetic, or isotonic strength could be taken depending on the available equipment and the patients' abilities. However, consensus among IMACS members was not reached on these tests (consensus scores ranged $40 \%$ to $55 \%$ ). Therefore, these tests were removed from the final candidate core set (Table 3).

Handgrip strength was included in the final candidate core set because the consensus scores were above the predefined $75 \%$ cutoff. However, because handgrip strength does not always identify postexercise changes in muscle performance, the MMT has been included in the core set ${ }^{1}$. The panel of experts advised that the valid and reliable protocol of Rider, et al be followed and that the described 8 muscle groups be tested using the Kendall 0-10 scale ${ }^{42}$.

Initially, the suggestion of the expert panel was to perform an additional test when an MMT score of $\geq 6$ is achieved in a particular muscle group. One could choose to test isometric, isokinetic, or isotonic strength for that muscle group as well because they may be a more sensible mode of assessment than MMT in patients with little to no severe muscle involvement. For isometric and isokinetic strength, this could be done with a dynamometer, and for isotonic strength, the panel advised to measure 1 or 10-15 repetition maximum. Some clinimetric properties have been found for the 1-kg arm lift test ${ }^{14}$, but the experts rated the 1 or $10-15$ repetition maximum higher, and this test was therefore selected instead. The choice of isometric, isokinetic, or isotonic strength appears to be dependent on the equipment available, the abilities of the patient, and the competencies of the clinician.

Even though consensus was not reached on these 3 
additional muscle strength tests, it would still be advisable to do one of these tests if an additional muscle strength test is needed. The low consensus scores of these 3 additional muscle strength tests could possibly be explained by the lack of information the respondents of the Internet survey received. The respondents were not aware that these 3 tests were only listed in addition to the handgrip strength and MMT, and were to be considered as an adjunct measure should a clinician need more insight into the patient's strength. Another explanation for these low consensus scores may be the limited availability of testing equipment in the centers.

There were no clinimetric properties available for the selected handgrip, isokinetic, and isotonic strength tests. Further research is needed to determine whether these muscle strength tests and corresponding assessment equipment are valid and reliable to use in patients with IIM.

Muscle function test. For muscle function, it is advised to perform the CMAS in children and the FI-2 in adults because both tests have been found to be valid and reliable in patients with IIM. Clinimetric properties have been demonstrated in the 30 -s chair stand test ${ }^{14}$, but this test was not included in the core set because it was found to be redundant after the inclusion of the CMAS and/or FI-2. Consensus scores of both the CMAS in children and the FI-2 in adults were high (both 95\%).

Limitations. One of the limitations of this Delphi was the fact that the IMACS members who participated in the final Delphi round did not have extensive experience with exercise physiologic studies and exercise tests. This could have biased the final list of tests toward those the panelists were familiar with, and not what might be the most appropriate tests for patients with IIM.

Recommendations for future research and clinical practice. Future clinical trials studying the effects of rehabilitation or exercise for patients with IIM are advised to incorporate the outcome measures listed in this core set, because this will facilitate the comparability between studies. A review on the efficacy of exercise training in patients with IIM reported that a large variety of outcome measures were used in the studies included, which impeded data pooling, and metaanalysis ${ }^{6}$.

Further, our current report identified a major gap in the knowledge regarding the clinimetric properties of many outcome measures in juvenile and adult patients with IIM. In the final candidate core set presented in this article, MMT, CMAS, and FI-2 are the only tests with good reliability and validity data. Therefore, more research in this area is warranted.

Availability of the tests in centers and the expertise needed to carry out these tests were not identified in this Delphi survey because of leading arguments to have a test included in the core set. However, for implementation in clinical practice and research, we advise researchers and clinicians to obtain experience in carrying out these tests before use.

We have presented a candidate core set of fitness and strength tests for patients with childhood and adult IIM. The core set will help standardize the conduct and reporting of clinical trials of exercise therapies, and assist practitioners in deciding which tests to use when assessing patients with IIM in the clinical setting. This will facilitate comparability of results across studies and clinical programs.

\section{ACKNOWLEDGMENT}

We thank Minal Jain and Joseph Shrader for their critical review of the manuscript.

\section{APPENDIX 1.}

List of study collaborators. International Myositis Outcome Assessment Collaborative Study Group (who have chosen to include their names): Luis Arboleya, Richard Barohn, Marco van Brussel, Hector Chinoy, Lorinda Chung, Robert Cooper, Mazen Dimachkie, Richard Finkel, Ignacio Garcia-De La Torre, Takahisa Gono, Thomas Griffin, Hidenaga Kawasumi, Raju Khubchandani, Ingrid Lundberg, Frank Mastaglia, Britta Maurer, Liza McCann, Merrille Needham, Marzena Olesinska, Nancy Olsen, Annet van Royen-Kerkhof, Lidia Rutkowska-Sak, Claudia Saad-Magalhaes, Adriana Sallum, Helga Sanner, Albert Selva-O'Callaghan, Clovis Silva, Vetrila Snejana, Yeong-Wook Song, Richard Vehe, Robert Wortmann.

\section{REFERENCES}

1. Rider LG, Werth VP, Huber AM, Alexanderson H, Rao AP, Ruperto $\mathrm{N}$, et al. Measures of adult and juvenile dermatomyositis, polymyositis, and inclusion body myositis: Physician and Patient/Parent Global Activity, Manual Muscle Testing (MMT), Health Assessment Questionnaire (HAQ)/Childhood Health Assessment Questionnaire (C-HAQ), Childhood Myositis Assessment Scale (CMAS), Myositis Disease Activity Assessment Tool (MDAAT), Disease Activity Score (DAS), Short Form 36 (SF-36), Child Health Questionnaire (CHQ), physician global damage, Myositis Damage Index (MDI), Quantitative Muscle Testing (QMT), Myositis Functional Index-2 (FI-2), Myositis Activities Profile (MAP), Inclusion Body Myositis Functional Rating Scale (IBMFRS), Cutaneous Dermatomyositis Disease Area and Severity Index (CDASI), Cutaneous Assessment Tool (CAT), Dermatomyositis Skin Severity Index (DSSI), Skindex, and Dermatology Life Quality Index (DLQI). Arthritis Care Res 2011;63 Suppl 11:S118-57.

2. Takken T, Spermon N, Helders PJ, Prakken AB, Van Der Net J. Aerobic exercise capacity in patients with juvenile dermatomyositis. J Rheumatol 2003;30:1075-80.

3. Takken T, van der Net J, Helders PJ. Anaerobic exercise capacity in patients with juvenile-onset idiopathic inflammatory myopathies. Arthritis Rheum 2005;53:173-7.

4. Alemo Munters L, Alexanderson H, Crofford LJ, Lundberg IE. New insights into the benefits of exercise for muscle health in patients with idiopathic inflammatory myositis. Curr Rheumatol Rep 2014;16:429.

5. Ponyi A, Borgulya G, Constantin T, Váncsa A, Gergely L, Dankó K. Functional outcome and quality of life in adult patients with idiopathic inflammatory myositis. Rheumatology 2005;44:83-8.

6. Habers GE, Takken T. Safety and efficacy of exercise training in patients with an idiopathic inflammatory myopathy - a systematic review. Rheumatology 2011;50:2113-24.

7. US Department of Health and Human Services. Physical activity and health: a report of the surgeon general. [Internet. Accessed October 16, 2015.] Available from: www.cdc.gov/nccdphp/sgr/pdf/sgrfull.pdf

8. Noonan V, Dean E. Submaximal exercise testing: clinical application and interpretation. Phys Ther 2000;80:782-807.

9. Verschuren O, Takken T, Ketelaar M, Gorter JW, Helders PJ.

Personal non-commercial use only. The Journal of Rheumatology Copyright (c) 2016. All rights reserved. 
Reliability for running tests for measuring agility and anaerobic muscle power in children and adolescents with cerebral palsy. Pediatr Phys Ther 2007;19:108-15.

10. Schmidt RC. Managing Delphi surveys using nonparametric statistical techniques. Decision Sciences 1997;28:763-74.

11. Verschuren O, Ketelaar M, Keefer D, Wright V, Butler J, Ada L, et al. Identification of a core set of exercise tests for children and adolescents with cerebral palsy: a Delphi survey of researchers and clinicians. Dev Med Child Neurol 2011;53:449-56.

12. Oddis CV, Rider LG, Reed AM, Ruperto N, Brunner HI, Koneru B, et al. International consensus guidelines for trials of therapies in the idiopathic inflammatory myopathies. Arthritis Rheum 2005;52:2607-15.

13. Stoll T, Brühlmann P, Stucki G, Seifert B, Michel BA. Muscle strength assessment in polymyositis and dermatomyositis evaluation of the reliability and clinical use of a new, quantitative, easily applicable method. J Rheumatol 1995;22:473-7.

14. Agarwal S, Kiely PD. Two simple, reliable and valid tests of proximal muscle function, and their application to the management of idiopathic inflammatory myositis. Rheumatology 2006;45:874-9.

15. Huber AM, Feldman BM, Rennebohm RM, Hicks JE, Lindsley CB, Perez MD, et al; Juvenile Dermatomyositis Disease Activity Collaborative Study Group. Validation and clinical significance of the Childhood Myositis Assessment Scale for assessment of muscle function in the juvenile idiopathic inflammatory myopathies. Arthritis Rheum 2004;50:1595-603.

16. Alexanderson H, Broman L, Tollback A, Josefson A, Lundberg IE, Stenstrom CH. Functional index-2: Validity and reliability of a disease-specific measure of impairment in patients with polymyositis and dermatomyositis. Arthritis Rheum 2006;55:114-22.

17. Alexanderson H, Del Grande M, Bingham CO 3rd, Orbai AM, Sarver C, Clegg-Smith K, et al. Patient-reported outcomes and adult patients' disease experience in the idiopathic inflammatory myopathies. Report from the OMERACT 11 Myositis Special Interest Group. J Rheumatol 2014;41:581-92.

18. Miller FW, Rider LG, Chung YL, Cooper R, Danko K, Farewell V, et al; International Myositis Outcome Assessment Collaborative Study Group. Proposed preliminary core set measures for disease outcome assessment in adult and juvenile idiopathic inflammatory myopathies. Rheumatology 2001;40:1262-73.

19. Rider LG, Giannini EH, Brunner HI, Ruperto N, James-Newton L, Reed AM, et al; International Myositis Assessment and Clinical Studies Group. International consensus on preliminary definitions of improvement in adult and juvenile myositis. Arthritis Rheum 2004;50:2281-90.

20. Ruperto N, Ravelli A, Pistorio A, Ferriani V, Calvo I, Ganser G, et al; Paediatric Rheumatology International Trials Organisation (PRINTO); Pediatric Rheumatology Collaborative Study Group (PRCSG). The provisional Paediatric Rheumatology International Trials Organisation/American College of Rheumatology/European League Against Rheumatism Disease activity core set for the evaluation of response to therapy in juvenile dermatomyositis: a prospective validation study. Arthritis Rheum 2008;59:4-13.

21. Omori C, Prado DM, Gualano B, Sallum AM, Sá-Pinto AL, Roschel $\mathrm{H}$, et al. Responsiveness to exercise training in juvenile dermatomyositis: a twin case study. BMC Musculoskelet Disord 2010;11:270.

22. Omori CH, Silva CA, Sallum AM, Rodrigues Pereira RM, Lúciade Sá Pinto A, Roschel H, et al. Exercise training in juvenile dermatomyositis. Arthritis Care Res 2012;64:1186-94.

23. Varjú C, Pethö E, Kutas R, Czirják L. The effect of physical exercise following acute disease exacerbation in patients with dermato/polymyositis. Clin Rehabil 2003;17:83-7.

24. Takken T, van der Net J, Engelbert RH, Pater S, Helders PJ. Responsiveness of exercise parameters in children with inflammatory myositis. Arthritis Rheum 2008;59:59-64.

25. Takken T, van der Net J, Helders PJ. The reliability of an aerobic and an anaerobic exercise tolerance test in patients with juvenile onset dermatomyositis. J Rheumatol 2005;32:734-9.

26. Florence JM, van der Ploeg A, Clemens PR, Escolar DM, Laforet P, Rosenbloom B, et al. T.P.1.01 Use of the 6 min walk test as an endpoint in clinical trials for neuromuscular diseases. Neuromuscul Disord 2008;18:738-9.

27. Buono MJ, Roby JJ, Micale FG, Sallis JF. Predicting maximal oxygen uptake in children: modification of the Astrand-Ryhming test. Pediatr Exerc Sci 1989;1:278-83.

28. Alexanderson H, Stenstrom CH, Lundberg I. Safety of a home exercise programme in patients with polymyositis and dermatomyositis: a pilot study. Rheumatology 1999;38:608-11.

29. Alexanderson H, Dastmalchi M, Esbjörnsson-Liljedahl M, Opava $\mathrm{CH}$, Lundberg IE. Benefits of intensive resistance training in patients with chronic polymyositis or dermatomyositis. Arthritis Rheum 2007;57:768-77.

30. Heikkillä S, Viitanen JV, Kautiainen H, Rajamäki T, Mäntyvuo P, Harju T. Rehabilitation in myositis: preliminary study. Physiotherapy 2001;87:301-9.

31. Johnson LG, Collier KE, Edwards DJ, Philippe DL, Eastwood PR, Walters SE, et al. Improvement in aerobic capacity after an exercise program in sporadic inclusion body myositis. J Clin Neuromuscul Dis 2009; $10: 178-84$.

32. Johnson LG, Edwards DJ, Walters SE, Thickbroom GW, Mastaglia FL. The effectiveness of an individualized, home-based functional exercise program for patients with sporadic inclusion body myositis. J Clin Neuromuscul Dis 2007;8:187-94.

33. Rider LG. Outcome assessment in the adult and juvenile idiopathic inflammatory myopathies. Rheum Dis Clin North Am 2002; 28:935-77.

34. Arnardottir S, Alexanderson H, Lundberg IE, Borg K. Sporadic inclusion body myositis: pilot study on the effects of a home exercise program on muscle function, histopathology and inflammatory reaction. J Rehabil Med 2003;35:31-5.

35. Chung YL, Alexanderson H, Pipitone N, Morrison C, Dastmalchi $\mathrm{M}$, Ståhl-Hallengren $\mathrm{C}$, et al. Creatine supplements in patients with idiopathic inflammatory myopathies who are clinically weak after conventional pharmacologic treatment: six-month, double-blind, randomized, placebo-controlled trial. Arthritis Rheum 2007; 57:694-702.

36. Harris-Love MO. Safety and efficacy of submaximal eccentric strength training for a subject with polymyositis. Arthritis Rheum 2005;53:471-4

37. Hicks JE, Drinkard B, Summers RM, Rider LG. Decreased aerobic capacity in children with juvenile dermatomyositis. Arthritis Rheum 2002; 47:118-23

38. Mathiesen P, Hegaard H, Herlin T, Zak M, Pedersen FK, Nielsen S. Long-term outcome in patients with juvenile dermatomyositis: a cross-sectional follow-up study. Scand J Rheumatol 2012;41:50-8.

39. Mathiesen PR, Orngreen MC, Vissing J, Andersen LB, Herlin T, Nielsen S. Aerobic fitness after JDM - a long-term follow-up study. Rheumatology 2013;52:287-95.

40. Nader GA, Dastmalchi M, Alexanderson H, Grundtman C, Gernapudi R, Esbjornsson M, et al. A longitudinal, integrated, clinical, histological and mRNA profiling study of resistance exercise in myositis. Mol Med 2010;16:455-64.

41. Ravelli A, Ruperto N, Trail L, Felici E, Sala E, Martini A. Clinical assessment in juvenile dermatomyositis. Autoimmunity 2006;39:197-203.

42. Rider LG, Koziol D, Giannini EH, Jain MS, Smith MR, Whitney-Mahoney K, et al. Validation of manual muscle testing and a subset of eight muscles for adult and juvenile idiopathic inflammatory myopathies. Arthritis Care Res 2010;62:465-72. 